\title{
Une lésion blanche kératosique isolée de la muqueuse buccale
}

\author{
Tommaso Lombardi', Jean-Pierre Carrel' ${ }^{2}$, Jacky Samson ${ }^{2, *}$ \\ ${ }^{1}$ Laboratoire d'Histopathologie buccale, École de Médecine dentaire, Genève, Suisse \\ ${ }^{2}$ Division de Stomatologie et Chirurgie orale, École de Médecine dentaire, Genève, Suisse \\ *jacky.samson@unige.ch
}

Reçu le 26 juillet 2012, accepté le 27 juillet 2012

Mots clés :

muqueuse buccale / lésion

blanche / réaction lichénoïde /

kératose réactionnelle exogène /

irritation chimique

Key words:

oral mucosa / leukoplakia /

lichenoid reaction / exogenous

reactive keratosis / chemical

irritation

\begin{abstract}
Résumé - Les lésions blanches kératosiques de la muqueuse buccale sont fréquentes et comportent de nombreuses étiologies. Les kératoses réactionnelles exogènes constituent le groupe le plus important et il existe de nombreuses substances chimiques pouvant entrainer une irritation de la muqueuse donnant naissance à une kératose réactionnelle. Cette irritation peut être secondaire à un médicament ou à une autre substance, parfois parce qu'il y a un mésusage. Dans le cas rapporté, la kératose était due au Sen-Sen, un produit à base de réglisse, vendu pour rafraîchir l'haleine.
\end{abstract}

Abstract - The keratotic white lesions of the oral mucosa are common and they have many etiologies. The exogenous reactive keratosis represent the most frequent group and there are many chemical agents which may provoke a mucosal irritation induicing a reactive keratosis. This irritation may be secondary to drugs or to other substances, perhaps because there is a misuse. In this report, we discribe a case of keratosis of the vestibular and gingival mucosa due to Sen-Sen, a breath freshener containing licorice.
Une patiente âgée de 64 ans est venue consulter sur les conseils de son chirurgien dentiste traitant pour une lésion blanchâtre, siégeant dans le vestibule à hauteur de 13-15, qui n'existait pas lors de la consultation de contrôle précédente un an auparavant. Cette patiente, sans antécédents médicochirurgicaux, est en bonne santé et ne prend aucun traitement. Elle n'a jamais fumé et elle consomme de l'alcool de façon très modérée.

L'examen montre qu'il s'agit d'une lésion kératosique bien limitée, occupant le fond du vestibule et débordant largement sur la fibro-muqueuse gingivale du voisinage. Dans le fond du vestibule, cette kératose plane, assez homogène, est parcourue par de fines crêtes parallèles. À la palpation, on perçoit bien ces reliefs mais la muqueuse a conservé sa souplesse. À côté de cette lésion qui attire l'œil, il existe une petite plage légèrement érythémateuse et une kératose discrète, homogène et sans limites nettes, qui prolonge la kératose vestibulaire sur la joue (Fig. 1).

Au total, il s'agit d'une lésion blanche kératosique isolée, sans étiologie évidente. Cette lésion pourrait constituer une réaction lichénoide mais la patiente ne prend aucun médicament et il n'y a pas de

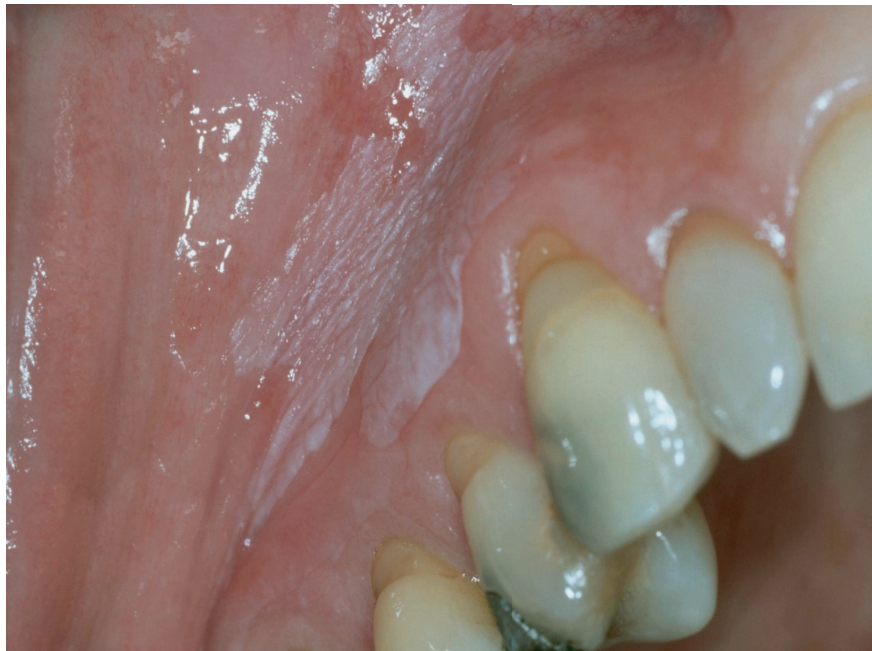

Fig. 1. Large plage kératosique isolée, asymptomatique, secondaire à l'application régulière d'une pastille de Sen-Sen.

Fig. 1. Wide leukoplakia, isolated and asymptomatic, secondary to the regular application of a lozenge of Sen-Sen.

contact avec un matériau susceptible de déclencher une réaction lichénoïde (amalgame...). La présence d'une plage érythémateuse qui occupe le centre de la lésion kératosique, peut faire suspecter un processus de transformation maligne, survenant exceptionnellement en l'absence de facteurs carcinogènes. Cette hypothèse étant peu probable, 
on doit envisager les autres étiologies des kératoses en commençant par les plus fréquentes, c'est-àdire les kératoses réactionnelles exogènes, avant d'envisager le diagnostic de kératose idiopathique. C'est en procédant ainsi, de façon systématique, que l'on apprend que la patiente place régulièrement, et plusieurs fois par jour depuis quelques mois, un comprimé de Sen-Sen dans son vestibule, toujours au même endroit, et qu'elle le laisse fondre sans le déplacer. L'arrêt de cette pratique a entrainé la disparition complète de la lésion en quelques mois.

\section{Commentaires}

Le Sen-Sen a été commercialisé aux USA à la fin du XIXe siècle sous forme de pastilles pour rafraichir l'haleine. Il est constitué de réglisse, de gomme arabique, de maltodextrine, de sucre et de parfums naturels et artificiels dont la composition reste confidentielle. Ce concurrent du Cachou Lajaunie est normalement bien toléré et, dans le cas de cette patiente, c'est le mésusage (laisser fondre la pastille sans la déplacer au lieu de la sucer et consommation abusive) qui est responsable de la kératose.

De nombreuses substances chimiques sont caustiques et peuvent entrainer une irritation, voire une brûlure de la muqueuse buccale lorsqu'elles restent suffisamment longtemps à son contact (aspirine, perborate de sodium, eau oxygénée, nitrate d'argent, acide trichloracétique, acide chromique, paraformaldéhyde, phénol, eugénol, hypochlorite de sodium... et de nombreux comprimés s'ils restent malencontreusement collés sur la muqueuse) $[4,5]$. C'est la répétition du contact prolongé ou le mésusage qui favorise l'apparition d'une kératose. Il y a quelques années, des produits pour l'hygiène bucco-dentaire (dentifrice, bains de bouche) contenant de la sanguinarine ou de la cannelle ont été commercialisés. Rapidement, il s'est avéré que leur utilisation régulière et prolongée entrainait l'apparition de plages kératosiques bien limitées sur la muqueuse gingivale et vestibulaire $[1,3]$; ils ont été retirés du commerce.
Devant une lésion blanche kératosique isolée de la muqueuse buccalechezunepatientesans antécédentsmédico-chirurgicaux, ne consommant pas de tabac et peu d'alcool, on peut écarter d'emblée de nombreuses étiologies. Il reste à envisager les diagnostics suivants : kératoses réactionnelles exogènes, réaction lichénoïde, kératose verruqueuse proliférante et kératose idiopathique. Le diagnostic de kératose verruqueuse proliférante est bien souvent tardif car, initialement, la lésion se présente comme une kératose simple, unique chez un sujet âgé, le plus souvent une femme, et touche surtout la gencive. La lésion s'étend progressivement, puis il en apparaît d'autres et la kératose prend peu à peu un aspect verruqueux. Au stade initial, la kératose verruqueuse proliférante ne présente cliniquement et histologiquement aucun caractère spécifique $[2,3]$. En l'absence de traitement médicamenteux, une éventuelle réaction lichénoïde ne pouvait être, en raison de sa localisation, que due à un contact prolongé et/ou répété avec un produit ou un matériau. La recherche de ce contact a conduit à la découverte du mésusage du Sen-Sen. La patiente ayant refusé la biopsie, il n'a pas été possible de trancher entre kératose réactionnelle et réaction lichénoïde mais sa disparition après avoir arrêté son utilisation laisse à penser qu'il est fort probable qu'il s'agissait d'une kératose réactionnelle.

Conflits d'intérêt : aucun

\section{Références}

1. Allen CL, Loudon J, Mascarenhas AK. Sanguinaria-related leukoplakia: epidemiologic and clinicopathologic features of a recently described entity. Gen Dent 2001;49:608-14.

2. Bagan J, Scully C, Jimenez Y, Martorell M. Proliferative verrucous leukoplakia: a concise update. Oral Dis 2010;16:328-32.

3. Kuffer R, Lombardi T, Husson-Bui C, Courrier B, Samson J. Lésions blanches kératosiques (pp. 55-67). In: La muqueuse buccale : de la clinique au traitement. Med'Com, Paris, 2009.

4. Laskaris G. Oral lésions due to chemical agents (pp. 66-75). In: Color atlas of oral diseases 3rd ed. Tieme, Stuttgart, 2003.

5. Neville BW, Damm DD, Allen CM, Bouquot JE. Epithelial pathology (pp. 388-97). In: Oral and maxillofacial pathology 3rd ed. WB Saunders Company, Philadelphia, 2009. 